\title{
NUOVA LUCE SU DEMOCRITO E LUCREZIO DA DIOGENE D'ENOANDA *
}

\begin{abstract}
New evidence on Democritos by Diogenes of Oenoanda concerning the theory of sleeping-images (fr. 1 and 6 and 13-12 Smith) permit to make clear some Democritos' known fragments (specially 55 A 77 Diels) and to interpret correctly some Lucretius' passages, e. g. 4, 788-801; 5, 1169-74; 4, 127 s.
\end{abstract}

Quando lo studio di un mondo frammentario come quello classico si accresce di nuovi documenti, ogni volta si concepisce la speranza di poter disporre di nuovi dati che permettano la soluzione di problemi aperti o almeno un approfondimento maggiore. Sotto questo aspetto non si puo certo dire che Democrito finora sia stato fra gli autori più fortunati. Nella storia dell'atomismo antico Epicuro e la sua scuola, durata così a lungo, hanno messo in ombra e portato quasi all'oblio tutti i predecessori, e della multiforme attività di Democrito solo opinioni sparse su singoli fenomeni della natura, o poco più, sono andate ad arricchire la dossografia. Non è rimasta nessuna esposizione continua e coerente della sua dottrina e non c'è stata ancora (né c'è da sperarlo) alcuna scoperta che riguardi direttamente quel filosofo, che pure esercito una grande influenza nello sviluppo del pensiero antico. E' quindi un felice caso che nelle recenti scoperte, fatte da M. F. Smith, della nota iscrizione murale di Diogene d'Enoanda si trovino due luoghi in cui si parla, con una certa ampiezza, di un'opinione di Democrito.

Essi ${ }^{1}$ sono venuti ad aggiungersi ai tre già noti di quell'iscrizione, relativi alla soggettività dei $\sigma \cup \mu \beta \varepsilon \beta \eta \kappa \delta ́ \tau \alpha$ per cui vien tolta validità

* Comunicazione presentata al Convegno Internazionale su «Democrito e l'antico materialismo», Catania, 18-21 aprile 1979.

1 Sono i fr. 1 e 6 pubblicati dallo Smith il primo in $A J A 74,1970$, pp. 56-58, il secondo ibid. 75,1971 , pp. 358-365. 
all' $\varepsilon v \nless \rho_{\gamma} \varepsilon\llcorner\alpha$ delle sensazioni (fr. $6 \mathrm{Ch} .=6 \mathrm{~W} .=7$ Gr., col. II, 2 ss.), al clinamen degli atomi ignorato da Democrito (fr. $32 \mathrm{Ch} .=33 \mathrm{~W} .=$ $30 \mathrm{Gr}$., col. II, 3 ss.), alla natura degli atomi (fr. 5 W. $=5$ Gr., col. II, 9 ss.). In quest'ultimo passo si dice che Democrito fece bene a porre come elemento base della natura gli atomi, a differenza di Eraclito, di Talete, di Diogene d'Apollonia e Anassimene, di Empedocle, di Anassagora, i quali ricorsero rispettivamente al fuoco, all'acqua, all'aria, ai quattro elementi, alle omeomerie; ma sbagliò in certi punti, come sarà mostrato in seguito. Ebbene le nuove testimonianze riguardano uno di questi punti e precisamente la natura delle visioni dei sogni. Si può dire in generale che viene approfondita la conoscenza di quel che sapevamo in proposito di Democrito da altre fonti e soprattutto che viene messo in rilievo un aspetto particolare di quell'argomento, che permette d'illustrare più di un passo di Lucrezio, in cui la critica ha errato nell'interpretazione o non ha visto il riferimento a Democrito.

Non è necessario che riporti il testo, ma rimando all'illustrazione dei nuovi frammenti di Diogene d'Enoanda che ho dato in Prometheus 3, 1977, pp. $2-13$ e $18-20$, dove, in particolare, ho dimostrato che il precedente fr. $7 \mathrm{Ch}$. (7 W., $8 \mathrm{Gr}$.) s'inserisce tra i nuovi fr. 6 e 1 Smith, di modo che, a partire dalla col. IV del fr. $5 \mathrm{Sm}$., si è ricuperata una lunga trattazione sulle immagini dei sogni, otto colonne più o meno complete. Mi limiterò a dare la traduzione dei brani che c'interessano.

Dopo aver esposta la teoria dei simulacri, di cui resta solo la conclusione nel nuovo fr. 5, I-III Sm. riguardante l'origine della vista e del pensiero e dei sogni, Diogene si sofferma a parlare dei sogni per confutare le opinioni in proposito degli Stoici e di Democrito. "Lo stesso, si dice in fr. 5, IV, 2 ss. e fr. 6, dobbiamo pensare dei simulacri che ci arrivano quando dormiamo. Allora affluiscono a noi ugualmente i simulacri e le impressioni, ma c'è differenza. Quando dormiamo, essendo tutti i sensi, per cosi dire, paralizzati e spenti nel sonno, l'anima, che resta sveglia 〈ma non è in grado di afferrare〉 la circostanza e la condizione di quel momento, nel ricevere i simulacri che le arrivano, concepisce di essi un'opinione non controllabile e falsa, come se essi fossero reali anche nella solidità. Infatti gli strumenti del controllo dell'opinione allora dormono e questi erano i sensi, nei quali soltanto stanno la regola e il criterio della verità contro 〈tutto ciò che è falso〉. Percio contro la tua dottrina, Democrito, diciamo questo: i sogni non sono affatto inviati dagli dei 〈allo scopo di spaventare o) ammonire, ma ciò che produce i sogni sono piuttosto certe impressioni, se almeno si tiene lontano il ragionamento sofistico.» 
Sul senso generale del passo non ci sono dubbi. Nell'autore è chiara la preoccupazione che ai sogni sia assegnata un'origine divina e che sia lasciata aperta la via alla divinazione, per cui egli dichiara subito «i sogni avvengono per incidenza di simulacri», come è detto nella Sent. Vat. $24^{2}$. Il termine usato da Diogene $\theta \varepsilon \delta \pi \varepsilon \mu \pi \tau$ o punto di vista epicureo ${ }^{3}$ e non aiuta a precisare il concetto che della divinità aveva Democrito; ma, in ogni modo, fa capire che per il filosofo quei simulacri erano collegati o collegabili col divino. In altre fonti (Vors. 55 A 78) si dice che Democrito chiamava «idoli i demoni e che l'aria ne è pienax, e Clemente Alessandrino (Str. V $88=$ II 383, 25 St. = 55 A 79) nota che anche Democrito avrebbe dovuto arrivare alla medesima conclusione di Senocrate che attribuiva qualche idea del divino persino agli animali, perché i suoi «idoli», che sono di sostanza divina

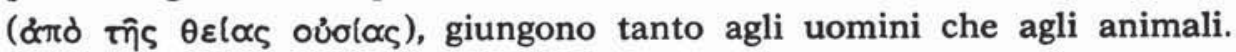
Secondo Cicerone (de nat. deor. I 12, $29=55$ A 74) Democrito pone fra gli dei ora le immagini e i loro tenui contorni ora la natura da cui quelle provengono. E sono chiamati divini anche i principia mentis, gli atomi dell'anima o intelletto che si trovano sparsi nell'universo in gran numero, e ancora certe immagini animate (animantes imagines) che sono solite giovare o nuocere agli uomini (ibid. 43, 120). Qui Cicerone addirittura dice deos i principia mentis, non diuina; ma ciò è dovuto al fatto che egli vuole rilevare la grande confusione che c'è in Democrito sulla natura degli dei, così da concludere, con grande sarcasmo, che quel filosofo, «giudicato fra i più grandi uomini che ci siano stati, è degno solo della sua patriax, è cioè stolto come gli altri Abderiti, i quali godevano fama di idioti ${ }^{4}$. Che poi di fronte alla stragrande quantità delle immagini dei sogni «di cui è piena l'aria si legga in Plinio (Nat. hist. II $14=55$ A 76) che Democrito ammetteva due divinità in tutto, la Pena e il Benefizio, mi pare una deduzione coerente, se si pensa che a quelle immagini appunto è attribuita la proprietà di giovare o di nuocere: nel primo caso il divino, in tutte le sue manifestazioni, si può chiamare Benefizio; nel secondo Pena. M. Wellmann ( $\propto$ Die Georgikà des Demokritos», Abhandl. d. Preuss. Akad., 1921, p. 15, n. 1) ha voluto attribuire quell'opinione al democriteo Bolo di Mende, neopitagorico, vissuto dopo il 200 a. C., e ciò può anche essere vero; ma

2 «I sogni non hanno sortito una natura divina né hanno una capacità divinatoria, ma avvengono per incidenza di simulacri».

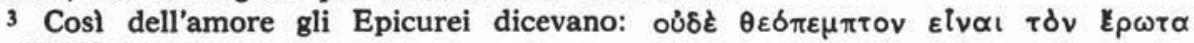
(fr. 574 Us.).

4 Cf. schol. Iuuen. X 33 e $47=55$ A 21; Cic., de nat. deor. I 43, $120=55$ A 74 . 
non c'è un reale motivo per negarla a Democrito e Bolo, se la professò, potrebbe averla derivata dalla concezione demologica di Democrito.

Più che di Dio o di dei con attributi specifici in Democrito si parlava del «divino», che riguardava l'aspetto superiore della costituzione atomica e cercava di accogliere e spiegare un'esperienza umana diffusa, accordando alle immagini dei sogni una forza attiva che le rende di lunga durata ma non immortali e che è capace d'influire favorevolmente o sfavorevolmente sull'uomo, il quale non può intervenire a stornarla con le preghiere. Egli qui è passivo e non può fare altro che «augurarsi d'imbattersi in simulacri di buon augurio (55 B 166). Siamo decisamente lontani da Epicuro che rivendicava la libertà assoluta dell'uomo, arbitro sempre di se stesso in forza della sua conoscenza e volontà. Al contrario Democrito ammetteva la possibilità di prevedere il futuro e considerava *cosa saggia l'abitudine degli antichi di scrutare le viscere delle vittime, perché si possono ricavare da esse degli indizi utili intorno alla salubrità e alle epidemie e talvolta anche sulla sterilità e fertilità dei campi», come c'informa Cicerone ( $D e$ diu. I 3, 5 e 57, $131=55$ A 138).

Non ci si dovrebbe quindi meravigliare se fra i libri di Democrito si trova un titolo come Sugli idoli o sulla previsione $(\pi \varepsilon \rho l \varepsilon l \delta \omega \lambda \omega \nu$ in rpovolins), che a torto è stato mutato dal Krische in Sugli idoli o

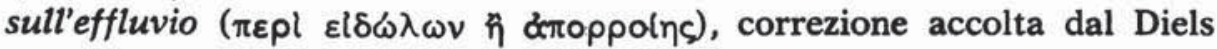
(55 B 10 a). Ma l'idea di «effluvio» sarebbe in pratica un'aggiunta inutile rispetto a quella di «simulacri», mentre il collegamento dei presagi con le immagini dei sogni è ricco di significato. Anche se nei passi citati di Cicerone si parla della previsione senza un accenno ai simulacri, non c'è da dubitare in proposito. Il filosofo voleva recare aiuto agli uomini evitando danni futuri, per cui insisteva in particolare sui simulacri malefici, «pieni del malefizio di quelli da cui sono emessi, che sconvolgono e danneggiano il corpo e l'animo con l'imprimersi e fissarsi e perdurare in coloro che sono colpiti» (Plut., Qu. Conu. V 7, 6, p. $682 \mathrm{~F}=55 \mathrm{~A} 77 \mathrm{a}$ ). Del resto la relazione fra previsione e idoli è esplicita nell'importante testimonianza di Sesto Empirico (Adu. Math. IX $19=55$ B 166): «Democrito dice che agli uomini si avvicinano dei simulacri, alcuni benefici altri malefici, per cui si augura d'incontrarne di propizi. Essi sono grandi e straordinari, resistono a lungo al disfacimento, ma non sono immortali, e predicono l'avvenire comparendo loro ed emettendo voci». Nelle ultime parole si accenna al modo in cui si pud compiere la previsione, ciò che Cicerone non dice, ma che ancor più chiaramente dे illustrato in una testimonianza, non meno importante, di Plutarco, a spiegazione del problema perché non si 
crede ai sogni d'autunno ( $Q u$. Conu. VIII 10, 2, p. $734 \mathrm{~F}=55 \mathrm{~A}$ 77). Favorino (test. 21 Bar.), si dice in quel luogo, riusci a chiarire un oscuro ragionamento di Democrito: «i simulacri penetrano profondamente in noi attraverso i pori e producono le visioni dei sogni. Essi affluiscono da ogni parte staccandosi dai mobili e dai vestiti e dalle piante e specialmente dagli esseri viventi, a causa della grande agitazione e del calore; e non solo possiedono la somiglianza delle forme del corpo da cui ricevono l'impronta - come dice Epicuro (fr. 362 Us.) che fino a questo punto segue Democrito ma l'ha abbandonato in seguito- ma assumono e tragono con sé anche le figure ( $z \mu \phi \not ̛ ́ c \mid c)$ dei movimenti e delle risoluzioni dell'anima e del carattere e delle passioni

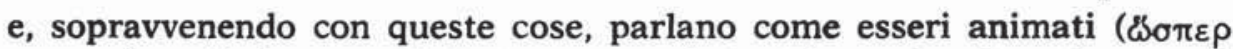
$\left.\varepsilon_{\mu \psi \cup \chi \alpha}\right)$ e rivelano a chi li riceve le opinioni ( $\left.\delta \delta \xi \propto \varsigma\right)$ e i ragionamenti ( $\delta\left\llcorner\alpha \lambda \circ \gamma\left\llcorner\sigma \mu \circ \varsigma_{\varsigma}\right)\right.$ e i desideri ( $\left.\delta \rho \mu \alpha \measuredangle \zeta\right)$ di quelli che emanano quei simulacri, a condizione che essi ci portino le immagini articolate e non confuse. E questo lo fanno particolarmente quando l'aria è calma, di modo che il tragitto avviene con rapidità e senza ostacoli».

Ho dato la versione dell'intera testimonianza (meno la chiusa in cui si dice che in autunno l'aria è più turbata e percid i simulacri arrivano meno compatti e meno vivaci), perché essa offre la spunto ad alcune considerazioni importanti, una relativa a Diogene d'Enoanda, altre a Lucrezio. Quel che Favorino dice di Democrito, che accordava alle visioni dei sogni la capacità di parlare e manifestare opinioni e ragionamenti e desideri, trova conferma nel nuovo fr. 1, col. II Sm. di Diogene. Questi, dopo aver replicato agli Stoici che non sono vuote le immagini dei sogni, ma hanno una natura corporea perché il vuoto non può essere riprodotto in immagini (fr. $7 \mathrm{Ch} .=7 \mathrm{~W} .=8 \mathrm{Gr}$.) mentre esse producono degli effetti reali, osserva contro Democrito: «Tuttavia,

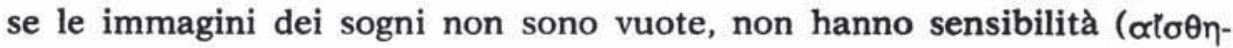

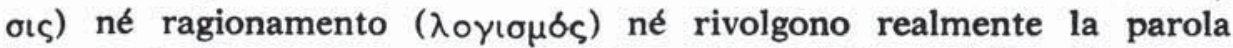
$(\pi p \circ \sigma \lambda \alpha \lambda \varepsilon \hat{i})$ a noi, come crede Democrito. E' impossibile che membrane cosı̀ sottili che non hanno la profondità di una natura solida abbiano queste proprietà. Costoro dunque hanno sbagliato in senso opposto, gli Stoici e Democrito: gli uni tolgono alle immagini dei sogni anche le capacità che hanno, Democrito regala loro quelle che non hanno.»

Propriamente nella testimonianza di Plutarco non c'è una parola precisa che indichi la sensibilità, ma questa è implicita in tutta l'illustrazione delle immagini che si comportano come esseri animati, e del resto di essa si parla esplicitamente nell'altro passo di Plutarco già citato a proposito del malefizio dei simulacri: «essi sono emanati da 
esseri malevoli e non sono affat to privi di sensibilità né di desiderio» $(Q u$. Conu. V 7, 6, p. 682 F = 55 A $77 \mathrm{a})$.

Perché poi Democrito accordasse la sensibilità alle immagini dei sogni, le quali sono, per quanto sottilissime, aggregati atomici, si deve forse al fatto che egli non riusciva a spiegare altrimenti gli effetti reali, spesso gravi, che i sogni hanno sugli uomini e gli animali. Non poteva spiegare la cosa ricorrendo ad un'eccitazione particolare dell'animo dei dormienti, secondo una teoria diffusa ${ }^{5}$, perché per quel filosofo il sonno è causato da insufficienza del soffio vitale ( $55 \mathrm{~A} \mathrm{136}$ ), in maniera analoga alla causa della morte, la quale avviene quando, nell'alterna espirazione e inspirazione degli atomi ignei e sferici che costituiscono l'anima, prevale l'espirazione perché la pressione dell'aria ambiente non riesce a respingere dentro gli atomi dell'anima cosicché si ha solo la fuga disastrosa di questi (55 A 106 e Leucippo 54 A 28). In maniera simile anche Epicuro spiegava il sonno attribuendolo ad un'espulsione parziale dell'anima del dormiente (Lucr. IV 907 ss.). In questo stato di passività, "quando i sensi tutti sono per così dire paralizzati e spenti nel sonno", come dice Diogene d'Enoanda nel primo passo tradotto, i simulacri compiono più facilmente la loro invasione e producono effetti più gravi; ma, mentre Democrito trasferisce alle immagini, oltre la forma, anche le proprietà dei senzienti, Epicuro, geloso custode e difensore della libertà umana, rileva l'incapacità dell'anima nel sonno, per quanto sia sveglia, di comprendere la situazione del momento e di giudicare rettamente, come ancora è detto nel medesimo passo di Diogene. Così Epicuro, coerente coi suoi principi gnoseologici, si ferma e non si lascia ingannare dalle apparenze e può correggere Democrito, che ha concesso troppe proprietà alle immagini dei sogni. Tutto questo è in perfetta consonanza con la spiegazione di Favorino riportata nel passo di Plutarco tradotto sopra.

$\mathrm{Da}$ quello che si è detto è lecito inferire, in linea generale, che anche in Lucrezio, quando si negano alle immagini dei sogni le proprietà dei senzienti, ci sia un riferimento specialmente a Democrito. Così in IV 788-801, nella sezione sull'origine del pensiero, il poeta indugia a chiarire alcune obiezioni, fra cui quella di simulacri che rappresentano persone in movimento:

Quid porro, in numerum procedere cum simulacra cernimus in somnis et mollia membra mouere, mollia mobiliter cum alternis bracchia mittunt et repetunt oculis gestum pede conuenienti?

5 Cf. Cic., De diu. II $58,120=55$ A 137 . 
scilicet arte madent simulacra et docta uagantur, nocturno facere ut possint in tempore ludos.

An magis illud erit uerum, quia...?

La spiegazione che segue, fondata sulla rapida successione delle immagini adatta a produrre un effetto "cinematografico", agevolato dal fatto che nei singoli tempi delle nostre percezioni ci sono molti attimi che restano non notati e che sono a disposizione simulacri d'ogni specie in qualunque momento pronti in ogni luogo, è contrapposta, non mi par dubbio, a Democrito, il quale attribuiva alle immagini dei sogni anche il movimento. Ciò è in pieno accordo col passo di Plutarco (55 A

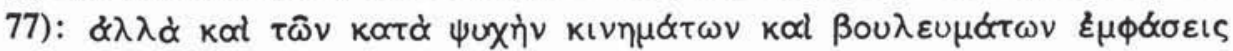

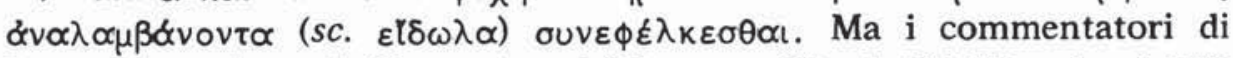
Lucrezio tacciono. Il Giussani anzi (Excursus II a 4, 720-819 nel vol. III, p. 290) trova "curioso» il passo di Plutarco e pensa che in esso «la dottrina di Democrito sia riferita con qualche esagerazione derisoria»; ma egli va cercando in quel passo elementi che possano chiarirgli un problema sottile e oscuro, quale il pensiero delle cose astratte, delle attività logiche, di cui non ci può essere alcun simulacro, e non ve lo trova. Ma la testimonianza di Plutarco, con la preziosa osservazione che Epicuro, pur accettando da Democrito la teoria dei simulacri, non giungeva ad accordare ad essi la proprietà del senziente come il movimento, è chiara e precisa, se è messa in rapporto col nuovo frammento di Diogene in cui è confutato Democrito per aver attribuito la sensibilità alle visioni dei sogni.

In un altro luogo Lucrezio, seguendo la tradizione della scuola epicurea, si serve della medesima dottrina di Democrito per spiegare l'origine della concezione volgare degli dei e della religione con le sue forme di superstizione, in particolare la divinazione. Dalla sezione 5, 1161-1240 il Diels ha estratto il gruppo di versi 1186-1193 (55 A 75a) per la corrispondenza di contenuto con la testimonianza di Sesto Empirico (Adu. Math. IX 24=55 A 75) relativa ai fenomeni celesti come lampi tuoni fulmini comete eclissi di sole e di luna, che sarebbero la causa, secondo Democrito, della credenza negli dei per il terrore che quei fenomeni incutevano negli uomini primitivi ${ }^{6}$. L'opinione era sicuramente anteriore a Democrito e divulgata nel mondo dei Sofisti. Un ampio frammento del Sisifo di Critia, conservato ancora da Sesto Empirico (Adu. Math. IX $54=$ fr. 25 Diels; I, p. $770 \mathrm{~N}_{2}$ ), dice che gli dei

6 Vedi K. Reinhardt, Hermes 47, 1912, p. 520 ss., dove è stato spiegato anche il passo di Clem. Al., Protr. $68=$ I 52, 16 St. e Str. V $103=$ II 394, 21 St. $=55$ B 30 , in rapporto con l'origine della religione. 
sono stati collocati nell'alto del cielo perché di li vengono i paurosi fenomeni meteorologici. Questo si accorda con Democrito e non ci sarebbero vere difficoltà cronologiche per ammettere un'eventuale derivazione di Critia dal filosofo; ma tutta l'argomentazione è presentata in rapporto col pensiero politico, che interessava soprattutto i Sofisti, che cioè sarebbe stato un uomo intelligente ad inventare la paura degli dei perché le leggi fossero osservate anche in segreto e si spegnesse con esse ogni violenza.

Sulle fonti del frammento di Critia sono stati espressi vari pareri ${ }^{7}$ e non è il caso di soffermarci, ma piuttosto di notare come sia opportuno aggiungere ai versi citati come testimonianza A 75 a di Democrito anche alcuni versi precedenti, 1169-1174:

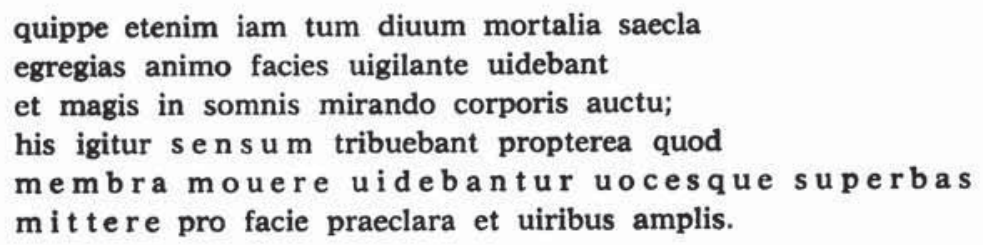

Il passo richiama in modo caratteristico il pensiero di Democrito, il quale accordava, come abbiam visto, la sensibilità, il movimento, la parola alle immagini divine che giungevano nel sonno. Nei versi che seguono (1175-1182) sono mescolati pensieri prettamente epicurei, come l'immortalità e felicità attribuita agli esseri divini, mentre per Democrito quelle imagines animantes, aggirantisi innumerevoli nell'aria, sia benefiche che malefiche, hanno solo una lunga durata ma non sono immortali (55 B 166). E la differenza appare dallo stesso Lucrezio. Infatti riguardo all'immortalità degli dei si dice che è la nostra mente ad inferire quella proprietà (putabant in 1168 e 1169), mentre in Democrito sono le immagini stesse che conservano le proprietà del senziente cosi da muoversi e parlare e le manifestano alla mente di chi sogna. Epicuro non poteva non negare la sensibilità anche ai simulacri divini, come ad ogni altro, operando un'importante correzione nella rappresentazione del divino di Democrito per salvare i suoi principi fisici e gnoseologici e etici, pur accettando da lui cose fantasiose e secondarie che non urtavano con quei principi, come la figura umana degli dei, di una grandezza straordinaria, con la sua attività di ragionare e parlare, naturalmente in lingua greca. Questa differenza appunto ci conferma che nei vv. 1169-1174 Lucrezio riferisce materia democritea.

7 Vedi A. Battegazzore, «Influssi e polemiche nel fr. $25 \mathrm{DK}$ di Crizia», Dioniso 21, 1958 , pp. $45-58$. 
Per Epicuro la negazione della sensibilità ai simulacri liberava l'uomo dal terrore superstizioso causato dal sogno di persone estinte che indurrebbero a credere nella sopravvivenza delle anime e nel loro aggirarsi tra i vivi. Per questo Lucrezio, nell'introdurre la teoria dei simulacri, sottolinea subito la sua importanza sul piano morale (IV 33 ss.), per salvaguardare l'atarassia in ogni momento, e in seguito (762-767) richiamerà l'attenzione sul principio che l'errore sta non nei sensi, ma nel giudizio e che nel sonno «tutti i sensi del corpo sono offuscati e riposano per cui non possono confutare il falso coi fatti e la memoria langue e non protesta obiettando che è già morta la persona che la mente crede di vederex. L'osservazione corrisponde esattamente a quello che dice Diogene d'Enoanda nel passo tradotto in principio (fr. 5-6 Sm.); ma non ci sarà nel poeta anche un accenno all'altra ragione per cui non si deve aver paura dei sogni, cioè la mancanza di sensibilità nei simulacri, che Diogene adduce nella medesima argomentazione (fr. $1 \mathrm{Sm}$.)? La cosa è tanto importante che difficilmente si può ammettere quel silenzio.

In perfetta armonia con Diogene suona una frase nel Contra Epicuri beatitudinem di Plutarco $(28,1105 \mathrm{E})$ a proposito dei simulacri degli amici morti, di cui gli Epicurei vogliono e credono vantaggioso conservare il dolce ricordo pur negandone la sopravvivenza: essi «non possie-

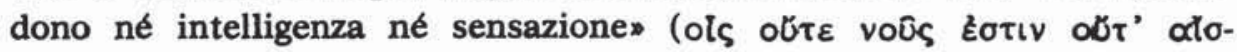

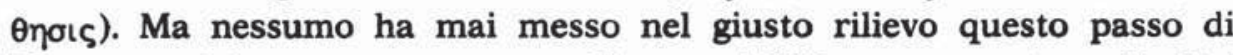
Plutarco né è stato estratto e collocato dall'Usener accanto agli altri concernenti gli eidola (fr. 317-320), ma è citato per intero solo come frammento di una lettera di Epicuro, da cui deriva, anche formalmente,

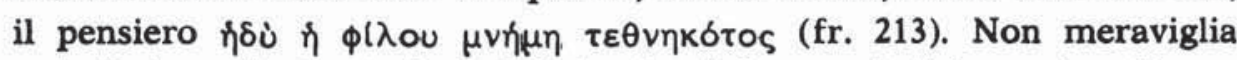
quindi che nella trattazione dei simulacri due versi di Lucrezio, riferentisi secondo me all'assenza in essi della sensibilità, non siano mai stati intesi esattamente, anche perché essi, che contengono la conclusione di un ragionamento, compaiono dopo una lacuna e non è chiara la loro connessione con cio che precede. Si tratta di IV 127 s.:

quin potius noscas rerum simulacra uagari multa modis multis nulla ui cassaque sensu 8.

Si sta ragionando sulla natura dei simulacri, tema che è cominciato nel v. 110, e precisamente sull'estrema sottigliezza che essi possiedono, vicina a quella degli atomi stessi. In quest'ordine di idee diventa im-

8 I due versi dipendono per la sintassi, secondo il Bailey, da un'espressione come «non c'è alcuna ragione perché tu non...». 
portante, anzi necessario, un accenno all'insensibilità dei simulacri. Ebbene io vedo un riferimento alla cosa nei due versi di quella conclusione.

Le parole nulla ui cassaque sensu hanno sempre procurato difficoltà agli interpreti, i quali vorrebbero intendere cassa sensu come «senza essere percepiti dal senso". Il Bailey, per rendere accettabile il valore passivo, parafrasa: «without the power of creating sensation»; ma sensus non eे «creazione d'una sensazione», bensì «la sensazione» 0 \&la capacità di sentire o percepire». Quindi la frase significa «privi di sensibilità», come in III 562 cassum anima corpus è "corpo privo di anima» $\mathrm{e}$ in IV 368 lumine cassus aer significa "l'ombra è aria priva di luce». Insomma la frase corrisponde a carentia sensu, precisamente come in II 990 carentibus undique sensu è detto degli atomi ${ }^{9}$, e appunto cassus è considerato il participio passato del verbo careo.

D'altra parte, nell'interpretazione tradizionale, bisognerebbe precisare che il senso di cui si parla è la vista, perché, se è vero che i simulacri sono così sottili che non sono percepiti da quell'organo, in realtà sono percepiti dal tatto, ché tutto nella fisica epicurea si riduce ad una sensazione tattile. Oppure bisognerebbe intendere, come suggerisce la parafrasi del Munro, che i simulacri non possono essere percepiti ad uno ad uno, ma che solo nella rapida successione continua producono la visione dell'oggetto. Ma in questo caso ci vorrebbe qualche altra parola, come singillatim in IV 89 e 105 nec singillatim possunt secreta uideri, ciò che è spiegato da Lucrezio più avanti, in IV 256 ss., col paragone del vento di cui non sentiamo ogni particella, ma tutte insieme esse ci fanno sentire qualcosa come di un corpo all'esterno.

Quanto all'altra frase nulla ui, qualcuno intende "senza forza», «senza effetto», un'anticipazione di cassa sensu, come il Munro: "they have no force and therefore are cassa sensu, can one by one make no impression on any of the senses». Ma il Giussani, per evitare la ripetizione, intese «senza corpo», «senza consistenza», dando a uis il valore di peso, quantità materiale, ed è stato seguito anche dal Bailey, che però non si mostra pienamente convinto per la ragione che il vocabolo in quel senso non è usato da Lucrezio. Ma il peso per Epicuro, come qualità primaria, non manca agli atomi e quindi ai simulacri, e rilevare che questi non hanno «alcun peso» sarebbe errato. D'altra parte l'interpretazione «senza forza», "senza efficacia» è equivoca, perché farebbe pensare che i simulacri non producono alcun effetto su chi li riceve, il

9 Cf. anche II 972 haud igitur debent esse ullo praedita sensu, sc. corpora prima. 
che non è vero. In IV 962 ss. Lucrezio si dilunga ad illustrare come si suole sognare qualcosa dell'attività giornaliera con effetti facilmente constatabili, e Diogene d'Enoanda, nelle prime due colonne del nuovo fr. $1 \mathrm{Sm}$. (insieme al fr. $7 \mathrm{Ch} .=7 \mathrm{~W} .=8 \mathrm{Gr}$., col. III, che, come ho dimostrato in Prometheus 3, 1977, p. 11 s., è la prima metà in senso verticale della col. I del nuovo frammento), mette in evidenza, per confutare l'opinione degli Stoici che le immagini dei sogni sarebbero vuote, come spesso si pena sognando qualche malattia o percosse e ferite o la caduta in un burrone, cosicché, spaventati, si balza su dal letto, e nota quel che succede al dormiente nel caso d'un sogno erotico.

Per me nulla ui significa «senz'alcuna qualità intrinseca» con riferimento alla mancanza delle proprietà del senziente, come il muoversi, il ragionare, il parlare, come appunto si dice dei simulacri dei sogni in Diogene d'Enoanda in opposizione a Democrito. L'uso di uis per indicare il valore intrinseco d'un oggetto è del tutto comune. In conclusione, il senso dei due versi è il seguente: «non vi è motivo perché tu non riconosca che molti simulacri vagano in molti modi e non possiedono alcuna qualità intrinseca e alcuna sensibilità».

Che ai simulacri, nella dottrina epicurea, siano negate le manifestazioni della sensibilità è un corollario del principio che gli atomi sono privi di senso, il quale è illustrato a lungo con vari argomenti da Lucrezio in II 865-1022, dopo la dimostrazione che gli atomi non possiedono le qualità secondarie. Infatti i simulacri sono così sottili che sono molto vicini alla struttura stessa degli atomi e non avendo la profondità di un solido è impossibile che possiedano le proprietà d'un senziente, come si afferma nel passo tradotto di Diogene. Epicuro avrà combattuto l'opinione opposta di Democrito nel libro secondo del $\Pi \varepsilon \rho l$

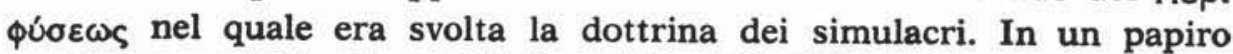
ercolanese resta la chiusa del libro (Pap. 993, 4 IV $=$ fr. 50, 19 II, 17 ss.

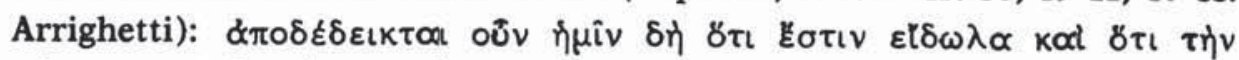

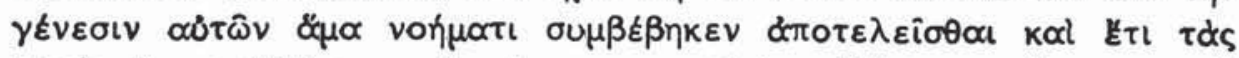

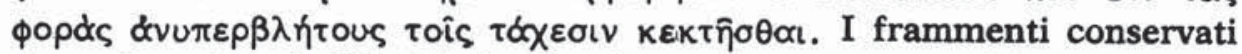
riguardano la velocità dei simulacri e conseguente capacità di superare rapidamente grandissime distanze mantenedosi inalterati ${ }^{10}$. Il problema di cui ci occupiamo sarà stato svolto in una precedente sezione sulla natura e rapidità con cui i simulacri si formano, due aspetti della teoria strettamente collegati tra loro ${ }^{11}$. In Lucrezio l'accenno all'assenza

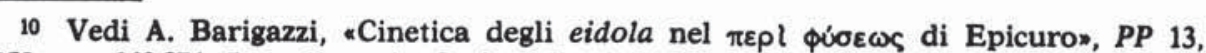
1958, pp. 249-276. I testi ora in G. Arrighetti, Epicuro. Opere, Torino 19732, p. 190 ss.

11 Nei frammenti ercolanesi superstiti potrebbe esserci qualche accenno al problema, ma lo stato del testo è cosi malconcio che non si pud affermare niente 
della sensibilità e di altre qualità nei simulacri, che abbiamo scoperto in IV $127 \mathrm{~s}$., si trova nel paragrafo iniziale sulla loro natura (IV 110144), a cui tien dietro il tema della velocità di formazione (145-175) e poi quello della velocità di spostamento (176-215), ciò̀ secondo la successione che s'incontrava nel libro di Epicuro.

Questa considerazione ci permette di trarre una deduzione interessante circa la lacuna in Lucrezio dopo il v. 126. Data l'importanza dell'argomento, essa con molta probabilità è discretamente ampia e il suo contenuto riguardava l'assenza nei simulacri delle qualità proprie d'un senziente, fors'anche con un richiamo all'ampia trattazione sulla mancanza della sensibilità nei singoli atomi in II 865-1022. E' un fatto che i due versi 127-8 si riferiscono a quell'argomento e non hanno un immediato aggancio con quel che precede.

A parte i nuovi passi di Diogene d'Enoanda, tra i frammenti di Democrito non ce n'è alcuno che illustri il pensiero del filosofo in proposito. Poiché anche per Democrito le qualità secondarie dipendono dalla forma posizione ordine degli atomi dentro un aggregato (55 A 49) e poiché anche i simulacri sono degli aggregati, si è subito disposti a credere che il filosofo abbia ragionato in maniera analoga a proposito delle proprietà del senziente. $O$ sarà da supporre che per Democrito la sensibilità non avesse origine dopo il «vortice», ma fosse una qualità innata di certi atomi singoli, ignei e sferici, quelli dell'anima o intelletto, con la caretteristica di muoversi e imprimere il moto per la loro peculiare sottigliezza e forma (55 A 74)?

Il problema dell'origine della sensibilità è di tale gravità che ha indotto alcuni dei materialisti moderni ad attribuire il senso agli atomi stessi, e in antico era usuale l'obiezione che la materia insensibile non può divenire sensibile e spiegare il pensiero. «E' già difficile, osserva Plutarco contro gli Epicurei (Adu. Col. 10, 1112 B = fr. 286 Us.), capire come si possano formare gli oggetti col movimento degli atomi; tanto meno si può concepire, anche con la migliore volontà, come dal vuoto e dagli atomi possano avere origine la sensazione e l'anima e l'intelletto e il pensiero, perché essi, presi separatamente, non hanno alcuna qualità e, mescolati, non subiscono alcuna affezione o mutazione e il loro incontro non porta ad una fusione o commistione o coalescenza, ma solo a urti e rimbalzi». Plutarco ripete obiezioni tradizionali dell'Acca-

con sicurezza e solo avanzare qualche ipotesi che possa servire in caso di ulteriori ritrovamenti. Per esempio, in fr. 41, $17 \mathrm{~V}$ Arr. si può supporre un pensiero come questo: «alle nature dei simulacri non si pud attribuire posizione e ordine di atomi, ma queste cose appartengono solo a quelle nature che in precedenza possedevano un'estensione». In fr. 47, 18 VI forse si accennava a Democrito e seguaci che accordavano al simulacro eun'estensione in profondità uguale agli oggetti solidi». 
demia, che hanno fatte proprie anche gli scrittori cristiani ${ }^{12}$; e dalla lunga trattazione di Lucrezio in II 865-1022, svolta con vari argomenti e non tutti chiari, si arguisce quanto fosse arduo rispondere in modo convincente agli avversari, sostenendo la dottrina di Epicuro che la percezione sensitiva è un prodotto della forma e posizione e ordine e moto degli atomi dentro un aggregato e mai fuori di esso, per cui un senziente è un composto di elementi non senzienti.

Ora i legami di Democrito col pensiero pitagorico e anassagoreo potrebbero far supporre che egli, pur cercando si sfruttare al massimo la concezione atomistica, conservasse qualcosa di quelle speculazioni per poter meglio spiegare certi fenomeni, come la sensibilità e il pensiero, che difficilmente si potevano conciliare con la riduzione di tutto a materia. Certamente Epicuro volle liberare gli atomi singoli da ogni qualità che non fosse primaria e spezzare decisamente ogni rapporto diretto fra il divino e l'umano. Ma tra Epicuro e Democrito ci fu di mezzo Aristotele, che acutamente chiarì la portata del pensiero di tutti i predecessori e inflù̀ non poco sulla costruzione atomistica, più limpida e coerente, di Epicuro, la quale poi si sostitul quasi totalmente all'opera di Democrito causandone la perdita.

In realtà, con la supposizione che Democrito attribuisse la sensibilità ai singoli atomi dell'anima o intelletto per la loro natura peculiare, si avrebbe una base più solida per ricostruire il suo pensiero psicologico e religioso, che sembra presentare residui di dottrine spiritualistiche, e di conseguenza quell'elevata morale o razionalismo ascetico che in un filosofo materialista ci sorprende, specialmente a confronto con il gretto utilitarismo epicureo, in quanto l'uomo mediante l'intelletto sarebbe partecipe di una forza primordiale che sola sarebbe in grado di portarlo alla conoscenza vera dell'universo in tutte le sue manifestazioni, cosicché il bene e il vero, che sono uguali per tutti gli uomini mentre diverso è il piacere (55 B 69), possono procurare quella serenità ed equilibrio che sono propri del saggio.

In favore della supposizione, qualcuno potrebbe essere tentato di addurre un passo di un nuovo frammento di Diogene d'Enoanda, proveniente dal trattato etico, il fr. $13-12 \mathrm{Sm} .{ }^{13}$. Vi si parla dei simulacri

12 Cf. Lact., Diu. inst. II 17, $29=$ fr. 287 Us. quo foedere inter se, qua mente conueniunt, ut ex iis aliquid conseratur, si sensu carent nec coire tam disposite possunt? quia non potest quicquam rationale perficere nisi ratio.

13 La numerazione è doppia perché il primo editore aveva considerato le due colonne indipendenti ( $A J A 75,1971$, pp. 376-381), ma poi si accorse che le due pietre, e quindi le due colonne, erano giustapposte con la successione inversa a quella data in precedenza (Thirteen New Fragments of Diogenes of Oenoanda, Oest. Ak. d. Wiss., Ergänzungsbände zu den Tituli Asiae Minoris Nr. 6, Wien 1974, pp. 4447).

XLIX, $10^{\circ}-2$ 
dei sogni per dimostrare come siano vane tutte le paure che quelli sembrano incutere, un punto della dottrina che naturalmente interessa da vicino il campo etico, come appare anche dai versi che Lucrezio premette alla trattazione della teoria in IV 26 ss. Richiamata la natura reale delle visioni nei sogni, contro la falsa opinione degli Stoici già combattuta nel trattato fisico, si aggiunge: $\tau \dot{\alpha}$ oûv $\varepsilon \nmid \delta \omega \lambda[\alpha / \tau] \alpha \hat{u} \tau \alpha$

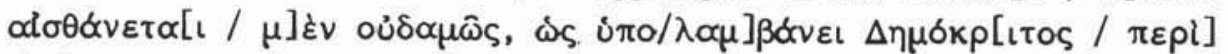

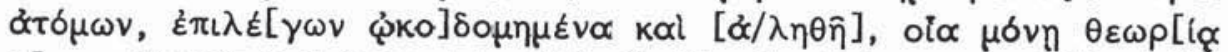

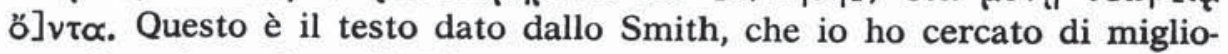
rare introducendo nell'ultima parte la correlazione con $\mu \varepsilon_{\nu}(\varepsilon \pi i \lambda \varepsilon[\pi \tau \alpha$

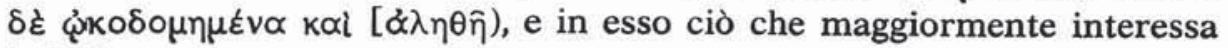

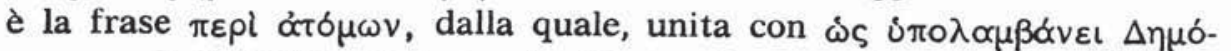
кpıtos, si dovrebbe dedurre che Democrito accordava la sensibilità a certi atomi (non sarebbe necessario pensare ad una lacuna $\pi \varepsilon \rho i ~ đ \alpha \tau \delta \mu \omega \nu$ $\langle\tau(v \omega ิ v\rangle)$, a quelli ignei e sferici dell'anima con cui il filosofo identificava l'intelletto (55 A 101), ai principia mentis sparsi per l'aria e divini (55 A 74 = Cic., de nat. deor. I 43, 120).

Ma quanto più grave è la deduzione, tanto maggiore dev'essere la prudenza. Il testo di Diogene non è sicuro e non può essere posto alla base di quell'interpretazione. Basta riferire la parola đó $\tau \dot{ } \mu \omega \nu$ agli atomi dei simulacri di cui si sta parlando e il pensiero del nuovo fr. 13-12 non differisce minimamente da quanto è stato osservato in precedenza nel trattato fisico, nel nuovo fr. 1 Sm., col. II, 10 - III, 6 o

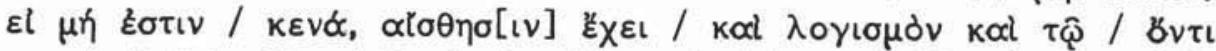

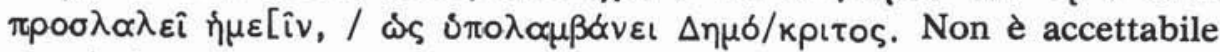
una lezione come $\pi \varepsilon \rho l \tau \hat{\imath} \nu$ ó $\tau \delta \dot{ } \mu \omega \nu$ "a proposito dei loro atomi», cioè degli atomi di quei simulacri, perché l'integrazione sarebbe troppo lunga e anche l'espressione sarebbe piuttosto ambigua, bastando $\pi \varepsilon \rho l$

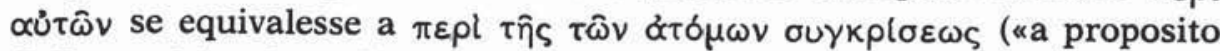
della combinazione di quegli atomi»). Penso che si debba porre virgola

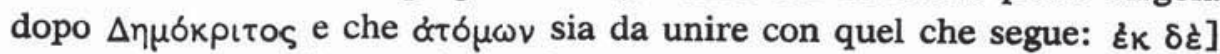

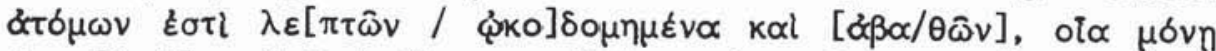
$\theta \varepsilon \omega \rho[l \mid \alpha b] \nu \tau \alpha^{14}$. Così c'è una perfetta corrispondenza con il precedente passo del trattato fisico, a cui nel nuovo fr. 13,8 ss. c'è, mi pare, un riferimento esplicito. Infatti in quel luogo alle righe riprodotte sopra si

14 Per motivo di spazio eventualmente ह̌k $\left.\delta^{\prime}\right]$; $\mathrm{mi}$ pare che l'originario $\varepsilon \pi \mathrm{L}$ sia stato corretto in $\varepsilon \sigma \tau \iota$, quindi non $\varepsilon \pi\lrcorner \lambda \varepsilon[\pi \tau \omega \nu$. Per ragioni di spazio qualcuno

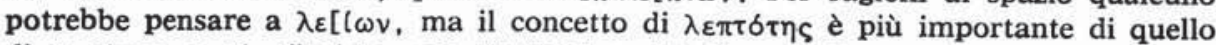

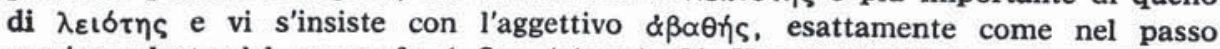

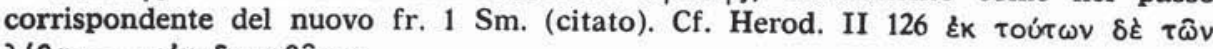

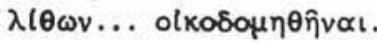




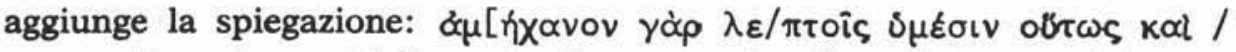

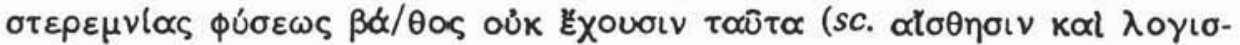

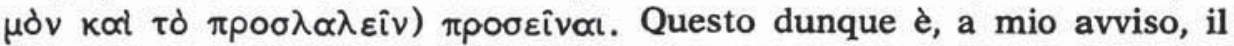
senso del passo in discussione: «questi simulacri dunque, quanto a sensibilità, non ne hanno affatto, come pensa Democrito, ma sono costruiti con atomi sottili e senza profondità, percepibili con la sola mente».

Adelmo BarigazzI 